\title{
The role of a multidisciplinary team in chest wall trauma management
}

\author{
Davide Patrini ${ }^{1}$, David Lawrence ${ }^{1}$, Savvas Lampridis ${ }^{1}$, Fabrizio Minervini ${ }^{2}$, Lorenzo Giorgi ${ }^{3}$, \\ Roberto Palermo ${ }^{3}$, Martin Hayward ${ }^{1}$, Marco Scarci ${ }^{4}$, Joachim Schmidt ${ }^{5}$, Benedetta Bedetti ${ }^{5}$ \\ ${ }^{1}$ Department of Thoracic Surgery, University College London Hospitals, London, UK; ${ }^{2}$ Department of Thoracic Surgery, Cantonal Hospital \\ Lucerne, Lucerne, Switzerland; ${ }^{3}$ Department of Thoracic Surgery, University of L'Aquila, L'Aquila, Italy; ${ }^{4}$ Department of Thoracic Surgery, San \\ Gerardo Hospital, Monza, Italy; ${ }^{5}$ Department of Thoracic Surgery, Malteser Hospital, Bonn, Germany \\ Contributions: (I) Conception and design: D Patrini, B Bedetti; (II) Administrative support: M Scarci; (III) Provision of study materials or patients: \\ M Hayward, J Schmidt; (IV) Collection and assembly of data: L Giorgi, R Palermo; (V) Data analysis and interpretation: D Patrini, B Bedetti, S \\ Lampridis; (VI) Manuscript writing: All authors; (VII) Final approval of manuscript: All authors. \\ Correspondence to: Mr. Davide Patrini. Consultant Thoracic Surgeon, Thoracic Surgery Department, University College London Hospitals, 16-18 \\ Westmoreland St. W1G 8PH, London, UK. Email: davide.patrini@nhs.net.
}

\begin{abstract}
Trauma represents the leading cause of death in patients younger than 45 years old. The introduction of multidisciplinary trauma teams has resulted in an improvement in patients`outcome. The thoracic surgeon plays an invaluable role as a member of the multidisciplinary team that includes an emergency physician, surgical figure, an anaesthetist, a radiology technician plus a number of nurses. Thorough knowledge of the physiological mechanism of injury and an intensive training have contributed massively in improving the outcomes. Different surgical approaches have to be considered in order to provide the best outcome to the acute patient with chest injury.
\end{abstract}

Keywords: Thoracic surgery; chest wall trauma; multidisciplinary team

Received: 11 November 2019; Accepted: 29 November 2019; Published: 05 April 2020.

doi: 10.21037 /jovs.2019.12.01

View this article at: http://dx.doi.org/10.21037/jovs.2019.12.01

\section{Introduction}

Despite substantial advances in trauma care over the last few decades, trauma still represents the leading cause of death in patients younger than 45 years old (1-3). Failure in providing sufficient specialized care in the early phase of major trauma led to the development of a modern model of civilian trauma system with the Emergency Medical System Act Public Law 93-155 issued by the United States Congress in 1973 (4). One of the major results from this law was the introduction of multidisciplinary trauma teams with the purpose to stabilize the patient and reduce the time span between the diagnosis and the treatment, with the only goal to improve overall survival. Several studies (5-8) have concluded that the inclusion of different specialties is essential to reduce mortality.

As effectively described by Ludwig et al. (9) management of chest trauma is based on three distinct levels of care ranging from prehospital trauma support, hospital trauma life support and surgical trauma life support. During the primary assessment of the trauma patient as per the Advanced Trauma Life Support (ATLS) (protocol, lifethreatening injuries should be excluded or managed. These include airway obstruction, pneumothorax, haemothorax, flail chest and cardiac tamponade. Subsequently, potentially life-threatening injuries should be dealt with or ruled out, including pulmonary and myocardial contusion, diaphragmatic injury and disruption of the tracheobronchial tree, oesophagus and aorta.

The thoracic surgeon plays an invaluable role as a member of an extended, multidisciplinary team, whose main goal is to reduce morbidity and mortality secondary to trauma. A good knowledge and profound understanding of the underlying pathophysiologic mechanisms associated with thoracic trauma is necessary to guide the management of this challenging clinical entity. 


\section{The concept and composition of the trauma team}

A team spirit is mandatory for appropriate delegation of tasks among members involved in the assessment and management of the trauma patient. In this context, a horizontal distribution of tasks has been proven to be effective in improving clinical outcomes (10). Regarding the composition of the trauma team, variations exist between different countries and healthcare system $(11,12)$; however, several factors are often common. In a basic set up, the trauma team is often led by a surgeon who coordinates according to the ATLS guidelines; his role can also be taken by an emergency physician. The rest of the team is composed by an anaesthetist, a radiology technician and a variable number of nurses. Airway management, which takes priority over all the other tasks, is usually carried out by the anaesthetist. His tasks also include intubation, ventilation and airway-associated interventions. The surgeon is responsible for the coordination of the trauma team, the primary survey of the patient and potential operative procedures. The radiology technician performs imaging investigations and may assist the surgeon in their interpretation. A radiologist will be required if a formal report is needed and in case the patient undergoes computed tomography (CT). Finally, the nursing staff make the alert calls, record vital information, take blood samples, place monitoring devices, set up the ventilator and assists the anaesthetist and the surgeon. It is worth noting that in some hospitals a neurologist or a neurosurgeon is often involved to assess more accurately the Glasgow Coma Scale score, focal neurological deficit and pupillary light response.

\section{Prehospital management}

Patient assessment with clinical examination including, inspection, palpation, percussion and auscultation are essential to recognize major thoracic injuries, such as open and tension pneumothorax, hemothorax, flail chest and lung contusion. Tension pneumothorax is the most frequent reversible cause of cardiac arrest (12). Therefore, rapid and accurate assessment for diminished chest expansion and absence of breath sounds on the ipsilateral side can be lifesaving. Needle decompression is the first line of treatment followed by insertion of a chest drain.

\section{Diagnostic imaging}

Patients with thoracic trauma are at high risk for both intrathoracic and intraabdominal injuries, depending on the mechanism and energy of the injury. Thorough assessment with various imaging modalities is advised in all but few cases, in which clinical suspicion is high and any delay in treatment may prove catastrophic. Imaging typically includes extended focused assessment with sonography in trauma (eFAST) for rapid detection of hemothorax, hemopericardium, pneumothorax and intraperitoneal bleeding $(13,14)$. More advanced imaging investigations include CT of the chest, which provides more detailed information. A CT is characterised by greater sensitivity and specificity compared to a plain chest radiograph, although the latter is generally sufficient to detect clinically significant injuries. However, a CT should be avoided in hemodynamically unstable patients. In these cases, the importance of eFAST to rule out life threatening injuries cannot be underestimated.

\section{Emergency room management}

A full assessment of the patient should be performed by the trauma team in the emergency room according to a predefined algorithm. Thorough and repeated clinical examination, review of the mechanism of thoracic injury and information about the past medical history of the patient are considered invaluable tools for successful management. According to the ATLS guidelines (15), a thoracic surgeon should be involved in case of persistent blood loss after chest drain insertion $(1,500 \mathrm{~mL}$ acutely or more than $200 \mathrm{~mL}$ per hour for 3-4 consecutive hours), severe subcutaneous emphysema, massive haemoptysis, penetrating chest trauma and substantial air leak from a chest tube. Immediate surgery is advised in case of persistent intrathoracic bleeding, endobronchial blood loss with ventilation impairment, penetrating injury and flail chest.

\section{Surgical management}

In order to access the chest in an emergency setting, anterolateral thoracotomy is the access of choice permitting good exposure of thoracic organs. Differently from the classic posterolateral thoracotomy a more anterior approach will avoid the rotation of the patient on a side. In $20 \% \mathrm{pf}$ patients the thoracotomy may be insufficient to visualize all the lesions therefore alternative approach such as Clamshell (transverse sternotomy and bilateral anterolateral thoracotomy) may be required (16). 
In the management of chest trauma there is also a role for minimally invasive surgery: indications for video assisted thoracoscopic surgery (VATS) may include (I) penetrating injury with minimal blood loss on a stable patient; (II) hemothorax; (III) empyema; (IV) persistent air leak; (V) concerns about diaphragmatic involvement.

VATS has shown its value in the management of pleural space involvement in the non-critical stable patient (17). Thoracoscopic assessment of the pleural cavity can demonstrate misdiagnosed injuries and lesion and treat a potential persisting hemothorax (18). Jin et al. (19) highlighted an advantage of VATS over open thoracotomy approach in a randomized trial demonstrating a lower rate of ARDS rates comparing to open thoracotomy patients.

The benefits of VATS are evident as long as strict inclusion criteria are respected: in a hemodynamically unstable patient with severe chest or cardiac vessel injury an open approach must be the preferred choice leaving no place for thoracoscopic surgery that can only have a negative impact on outcomes by delaying the unavoidable conversion to open thoracotomy (20).

\section{The effect of the trauma team on clinical outcome}

The aim of the trauma team is to reduce morbidity and mortality and thus improve patient outcome. Indeed, since the introduction of the trauma team worldwide, there is strong evidence to support that patient outcome has improved significantly (21). This improvement is noted not only in the improved management of moderately and severely injured patients, but also in the higher rate of unexpected survivors due to the efficient role of the trauma team. Contrariwise, patients who meet the criteria for trauma call and are not treated by a trauma team, demonstrate higher rates of morbidity and mortality (22).

\section{Trauma team training}

\section{Advance trauma life support course (ATLS)}

The first ATLS course was held in USA in 1978. It was originally developed for doctors in rural areas and subsequently expanded by the American College of Surgeons in 1980. The ATLS course is now widely accepted and has resulted in better patient outcomes in several studies (23).

\section{Simulators}

Simulation-based training creates a situation where certain skills are applied as in a real-world environment. Skill development is achieved through repetition and constructive feedback (24). There are several modalities of simulation-based training, which all share the same goal: to enable the trainee to acquire a wide set of skills. There is no consensus whether using a standardized manikin or a patient will make a difference when considering communication, cooperation and leadership skills (25). A study by Wisborg and colleagues (26) confirmed this observation, although considering that the outcome is measured in participant's assessment of their role and the degree of realism rather than with a more objective outcome score.

\section{Videotaping}

Several studies have been published on the benefits of recording simulated or actual trauma situations (27-29). The benefits of this are threefold: firstly, recorded video can be used for educational purposes, by creating opportunities to review and modify behaviour in a controlled environment; secondly, it can be used to assess and measure the adherence to the ATLS protocol; lastly, recorded video can be used also for research purposes (30).

\section{Conclusions}

Leadership and communication skills are considered of paramount importance in the effective management of a trauma team. Moreover, appropriate supervision and support by the senior members, as well as self-awareness and ability to seek help by the junior members, are warranted to improve the efficacy of the team. Furthermore, thorough knowledge of the role of each member and clearly defined tasks may have a significant impact on the clinical outcome of trauma patients. Management of these patients should follow general principles of well-established trauma protocols and, depending on the pathophysiology of each individual case, it can range from simple observation to salvage surgery (Video 1). 


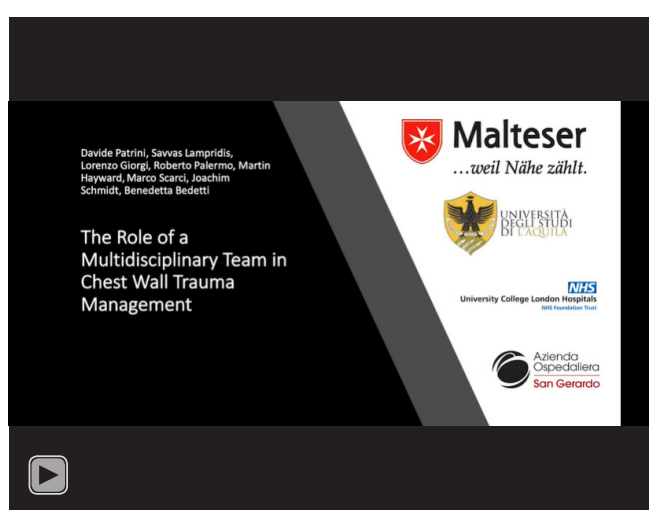

Video 1 Learning points in chest wall trauma management (31).

\section{Acknowledgments}

Funding: None.

\section{Footnote}

Provenance and Peer Review: This article was commissioned by the editorial office, Fournal of Visualized Surgery for the series "Chest Wall Traumas". The article has undergone external peer review.

Conflicts of Interest: All authors have completed the ICMJE uniform disclosure form (available at https://jovs. amegroups.com/article/view/10.21037/jovs.2019.12.01/ coif). The series "Chest Wall Traumas" was commissioned by the editorial office without any funding or sponsorship. FM served as the unpaid Guest Editor of the series. MS served as the unpaid Guest Editor of the series and serves as an unpaid editorial board member of fournal of Visualized Surgery from March 2019 to February 2021. The authors have no other conflicts of interest to declare.

Ethical Statement: The authors are accountable for all aspects of the work in ensuring that questions related to the accuracy or integrity of any part of the work are appropriately investigated and resolved.

Open Access Statement: This is an Open Access article distributed in accordance with the Creative Commons Attribution-NonCommercial-NoDerivs 4.0 International License (CC BY-NC-ND 4.0), which permits the noncommercial replication and distribution of the article with the strict proviso that no changes or edits are made and the original work is properly cited (including links to both the formal publication through the relevant DOI and the license). See: https://creativecommons.org/licenses/by-nc-nd/4.0/.

\section{References}

1. Centers for Disease Control and Prevention. Available online: www.cdc.gov

2. Central Bureau of Statistics of Netherlands. Available online: www.cbs.nl/en-gb

3. Norton R, Kobusingye. Injuries. N Engl J Med 2013;368:1723-30.

4. Harvey JC. The Emergency Medical Service Systems Act of 1973. JAMA 1974;230:1139-40.

5. West JG, Trunkey DD, Lim RC. Systems of trauma care. A study of two counties. Arch Surg 1979;114;455-60.

6. West JG, Coles RH, Gazzaniga AB. Impact of regionalization. The Orance County Experience. Arch Surg 1983;118:740-4.

7. Clemmer TP, Orme JF. Outcome of critically injured patients treated at level 1 trauma centers versus full-service community hospitals. Crit Care Med 1985;13:861-3.

8. Shatney CH. Initial Resuscitation and assessment of patients with multisystem blunt trauma. South Med J 1988;81:501-6.

9. Ludwig C, Koryllos A, Management of Chest Trauma. Journal of Thoracic Disease 2017;9:S172-7.

10. Liberman M, Mulder DS, Jurkovich GJ. The Association between trauma system and trauma center components and outcomes in a mature regionalized trauma system. Surgery. 2005:137:647-58.

11. Driscoll PA, Vincent CA. Organizing an efficient trauma team. Injury 1992;23:107-10.

12. Mistry N, Bleetman A, Roberts KJ. Chest decompression during the resuscitation of patients in prehospital traumatic cardiac arrest. Emerg Med J 2009;26:738-40.

13. Röthlin MA, Nöf R, Amgwerd M. Ultrasound in blunt abdominal and thoracic trauma. J Trauma 1993;34;488-95.

14. Kirkpatrick AW, Sirois M, Laupland KB, et al. Handheld thoracic sonography for detecting post traumatic pneumothoraces, the extended focused assessment with sonography for trauma (eFAST). J Trauma 2004;57:288-95.

15. ATLS Subcommittee., American College of Surgeons' Committee on Trauma., International ATLS working group. Advanced trauma life support (ATLS®): the ninth edition. J Trauma Acute Care Surg 2013;74:1363-6.

16. Karmy-Jones R, Nathens A, Jurkovich G, et al. Urgent and emergent thoracotomy for penetrating chest trauma. J 
Trauma 2004;56;664-8.

17. Schots JP, Vissers YI, Hulsewe KW, et al. Addition of Video-Assisted Thoracoscopic Surgery to the Treatment of Flail Chest. Ann Thorac Surg 2017;103:940-4.

18. Chou YP, Lin HL, Wu TC. Video-Assisted thoracoscopic surgery for retained hemothorax in blunt chest trauma. Curr opin Pulm Med 2015;21:393-8.

19. Jin J, Song B, Lei YC, et al. Video-Assisted thoracoscopic surgery for penetrating chest trauma. Chin J Traumatol 2015;18:39-40.

20. Mizobuchi T, Iwai N, Kohno H, et al. Delayed Diagnosis of traumatic diaphragmatic rupture. Gen Thorac Cardiovasc Surg 2009;57:430-2.

21. Petrie D, Lane P, Stewart TC. An Evaluation of patient outcome comparing trauma team activated versus trauma team not activated using TRISS analysis. Trauma and Injury Severity Score. J Trauma 1996;41:870-3.

22. Rainer TH, Cheung NK, Yeung JH, Graham CA. Do Trauma Teams make a difference? A single centre registry study. Resuscitation 2007;73:374-81.

23. Carmont MR. The Advanced Trauma Life Support course: a history of its development and review of related literature. Postgrad Med J 2005:81:87-91.

24. Shapiro MJ, Morey JC, Small SD, et al. Simulation based teamwork training for emergency department staff: doesit improve clinical team performance when added to an

doi: 10.21037/jovs.2019.12.01

Cite this article as: Patrini $\mathrm{D}$, Lawrence $\mathrm{D}$, Lampridis S, Minervini F, Giorgi L, Palermo R, Hayward M, Scarci M, Schmidt J, Bedetti B. The role of a multidisciplinary team in chest wall trauma management. J Vis Surg 2020;6:19. existing didactic teamwork curriculum? Qual Saf Health Care 2004;13:417-21.

25. Capella J, Smith S, Philip A, et al. Teamwork Training Improves the clinical care of trauma patients. J Surg Educ 2010;67:439-43.

26. Wisborg T, Braettebo G, Brinchmann Hansen A, Hansen KS. Mannequin or standardized patient: participants' assessment of two training modalities in trauma team simulation. Scand J Trauma Resusc Emerg Med 2009:17:59.

27. Santora TA, Trooskin SZ, Blank CA, et al. Video assessment of trauma response: adherence to ATLS protocols. Am J Emerg Med 1996;14:564-9.

28. Hoyt DB, Shackford SR, Fridland P, et al. Video recording trauma resuscitations: on effective teaching technique. $\mathrm{J}$ Trauma 1988;28:435-40.

29. Blank-Reid CA, Kaplan LJ. Video recording trauma resuscitation: a guide to system set up, personnel concerns and legal issue. J Trauma Nurs 1996;3:9-12.

30. Scherer LA, Chang M, Meredith JW, Battistella FD. Video tape review leads to rapid and sustained learning. Am J Surg 2003;185:516-20.

31. Patrini D, Lawrence D, Lampridis S, et al. Learning points in chest wall trauma management. Asvide 2020;7:044. Available online: http://www.asvide.com/watch/33084 